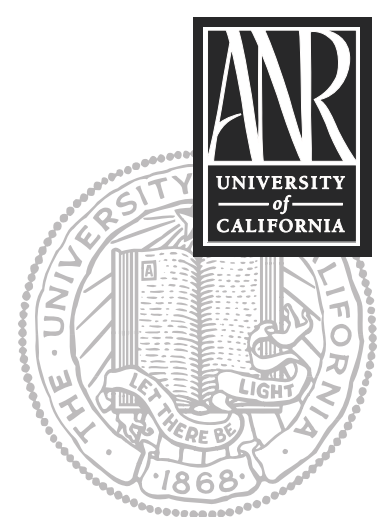

UNIVERSITY OF CALIFORNIA

Division of Agriculture and Natural Resources http://anrcatalog.ucdavis.edu

\title{
Selecting Fruit, Nut, and Berry Crops for Home Gardens in San Mateo and San Francisco Counties
}

K. S. JONES, UC Cooperative Extension Horticulture Program Representative, San Mateo-San Francisco Counties; and LAURENCE R. COSTELLO, UCCE Environmental Horticulture Advisor, San Mateo-San Francisco Counties

Although many fruit, nut, and berry crops can be grown in San Mateo and San Francisco Counties, not all crops can be grown successfully in all areas of the two counties. For crops to perform well in your location, they have to be selected carefully. This publication provides an extensive list of crops with an assessment of their suitability for three different climate zones in San Mateo and San Francisco Counties, and includes specific notes for each of the crops listed. With this information, you should be able to select the fruit, nut, and berry varieties best suited to your location.

\section{SELECTING CROPS AND VARIETIES: A TWO-STEP PROCESS}

To select crops (and crop varieties) for your location, follow this two-step process:

- Determine your climate zone from the "Climate Zones for San Mateo and SF Counties" section below. Using descriptions of the three zones, and taking microclimate factors into consideration (see "Other Requirements for Fruit Production" below), identify which zone is the best fit for your location.

- Choose crops and varieties from the "Crop and Variety Selection Table" located at the end of this publication, using assessments (yes, no, and marginal) given for each of the climate zones. Assessments are based largely on chill hours estimated for the climate zone and the chill requirement of the crop and its varieties. For information on chilling, see "All About Chill Hours" below. Be careful to select only those varieties with chill requirements that will be satisfied at your location. For instance, in Zone $C$ only select varieties with low chill requirements.

Keep in mind that crop varieties have specific characteristics, particularly in terms of fruit traits. Of the 14 apple varieties listed, each differs with regard to fruit color, size, shape, and flavor. Refer below to "Notes on Crops and Varieties" and other publications (see "Resources") for more information about specific varieties. Note that although many named varieties are listed in the table, not all available varieties are included.

\section{CLIMATE ZONES IN SAN MATEO AND SAN FRANCISCO COUNTIES}

Since climatic conditions vary considerably across San Mateo and San Francisco Counties, three climate zones have been identified. These zones are delineated largely by differences in summer and winter temperatures: Zone A has warm to hot summers and cool winters, Zone B has warm summers and mild winters, and Zone C has mild summers and winters. 


\section{Zone A}

Roughly equivalent to Sunset Zones 14 to 16, and includes San Carlos, Redwood City, Portola Valley, Woodside, Atherton, Menlo Park, East Palo Alto, and La Honda. Characterized by hot summers and cool winters, Zone A is considered climatically favorable for fruit, nut, and berry production. Summers are long and hot enough for maturation of most fruits. Some frost protection may be needed in the coldest locations in the zone. All varieties, except those with very high chill requirements (over 1,000 hours), can be expected to perform well.

\section{Zone B}

Roughly equivalent to Sunset Zone 16, and includes Millbrae, San Mateo, Hillsborough, Burlingame, Belmont, and Foster City. Warm summers and mild to cool winters characterize this zone. Moderate-chill (400 to 700 hours) or low-chill (less than 400 hours) varieties should be selected. Early- or midseason-ripening varieties do well.

\section{Zone C}

Equivalent to Sunset Zone 17, and includes San Francisco, South San Francisco, Brisbane, Colma, Daly City, San Bruno, Pacifica, Half Moon Bay, and San Gregorio. Coastal fog and wind dominate much of this zone in the summer months. Variety selection is limited in Zone $\mathrm{C}$ because of the characteristically moderate summers and

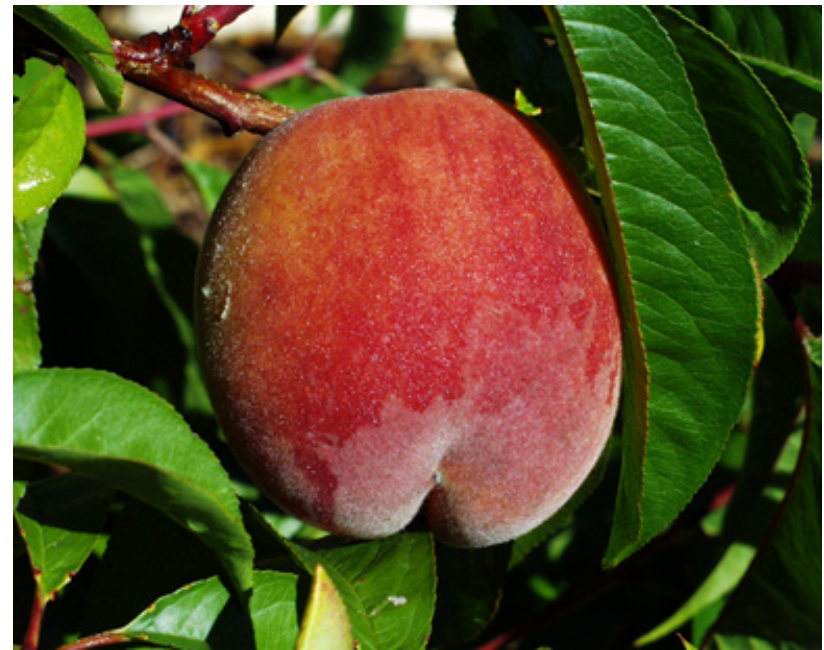

Figure 1. Peach is a temperate zone crop.

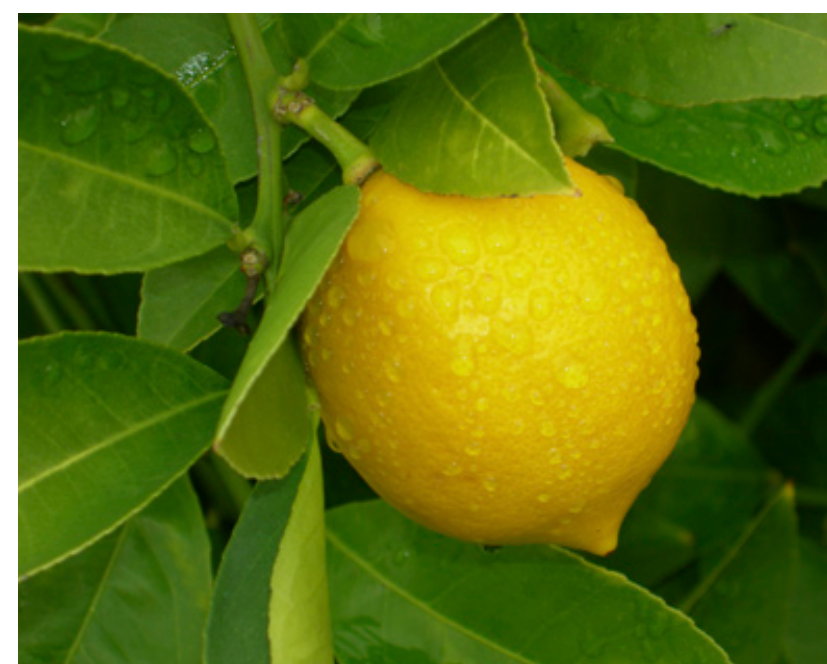

Figure 2. Lemon is a subtropical zone crop. winters. Too little winter chill and not enough summer heat prevents proper fruit development of many varieties. Low-chill (less than 400 hours) and early-ripening varieties should be selected.

\section{CROP TYPES: TEMPERATE, SUBTROPICAL, AND TROPICAL}

Fruit, nut, and berry crops are commonly grouped into one of three categories: temperate, subtropical, and tropical. Temperate zone crops include almond, apple, apricot, peach, grape, blueberry, and strawberry (fig. 1). Avocado, citrus, and guava are considered to be subtropical, while banana, cashew, and pineapple are tropical (fig. 2).

Generally, temperate and subtropical crops can be grown in San Mateo and San Francisco Counties (with some caveats), but tropical crops are rarely successful. This publication focuses on temperate and subtropical crops.

Temperate zone crops generally require a period of cold temperature during the winter months (from $32^{\circ}$ to $45^{\circ} \mathrm{F}$, or $0^{\circ}$ to $7.2^{\circ} \mathrm{C}$ ) for successful flower and fruit development. This cold temperature period is measured in "chill hours" (see "All About Chill Hours" below). Some crops require many chill hours, while others require few. This is called the crop's "chill requirement." When selecting temperate zone crops, it is important to choose only those crops that have a chill requirement that will be met at your location.

Subtropical crops, such as citrus, loquat, and guava, require little or no chilling. Native to warm-climate regions, these crops can be injured by cold temperatures 
during winter and spring months, and they require heat during the growing season for fruit maturation and flavor.

\section{OTHER REQUIREMENTS FOR FRUIT PRODUCTION}

Selecting climate zones and meeting chill requirements are not the only factors necessary for good fruit production. Pollination, sunlight, heat accumulation, and wind are all important considerations.

For fruit development to occur, flowers have to be pollinated; that is, pollen must move from the male organs (stamens) to the female organs (carpels). Pollen transfer can be facilitated by bees, beetles, flies, butterflies, moths, birds, bats, wind, and water. The pollen can come from flowers on the same tree (self-fertile), or from flowers of other trees of the same species (cross-pollination). In some cases, a crop may require another tree of a specific variety for pollination. For information on pollination requirements, see the "Crop and Variety Selection Table" at the end of this publication. For further details, see "Notes on Crops and Varieties" below. Note that even in selffertile crops, cross-pollination can increase fruit set. Also, poor pollination can occur

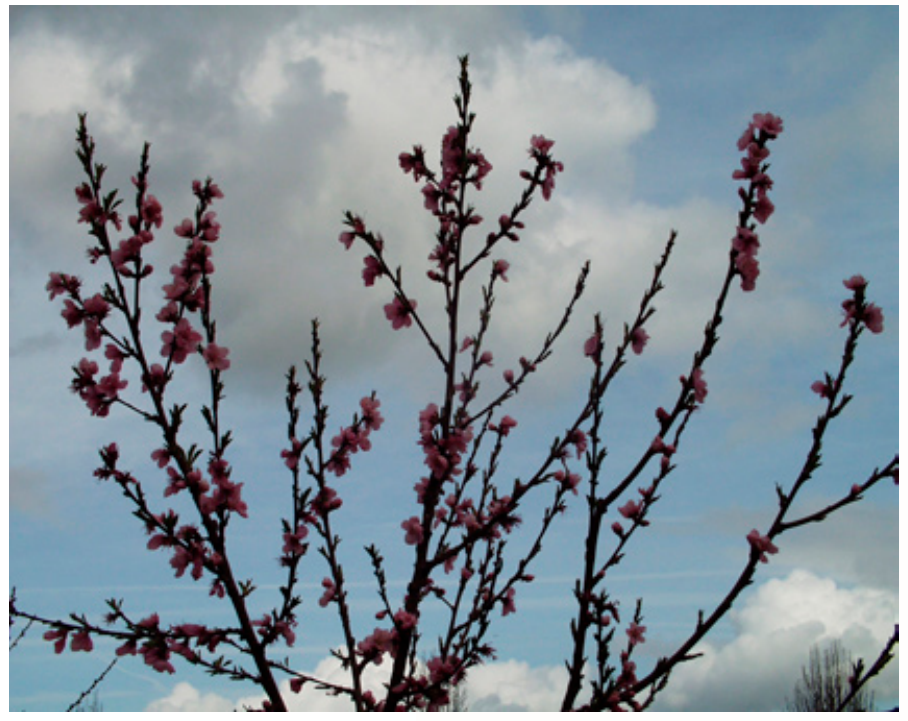

Figure 3. Cold and rainy spring days can lead to inadequate pollination.

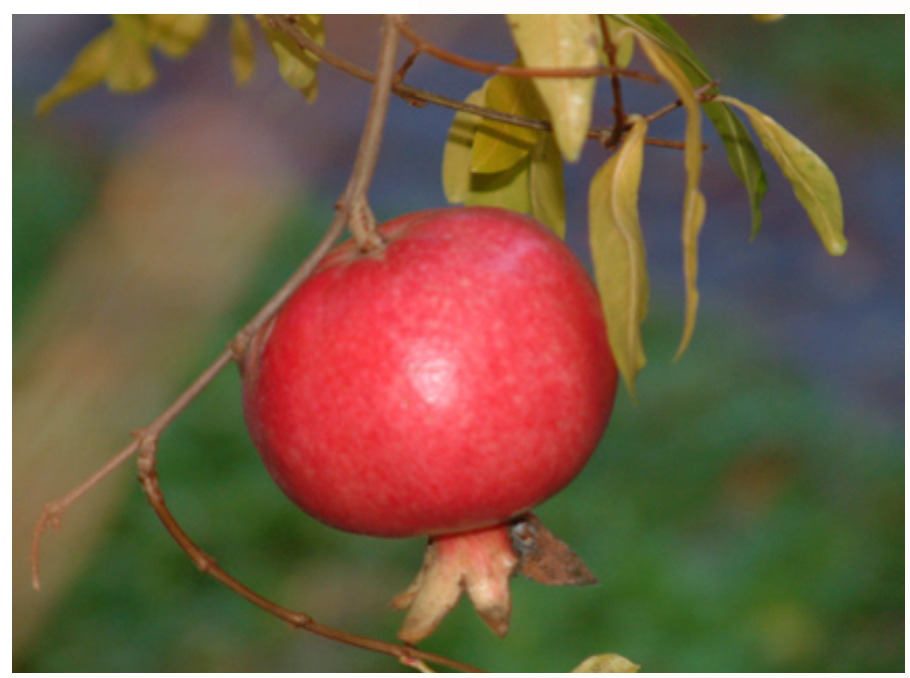

Figure 4. Pomegranate requires heat during the growing season for optimum fruit production. as a result of insufficient pollinator activity, such as during cool and wet weather in the spring when bees may be less active (fig. 3).

For most crops, plentiful sunlight (at least 6 hours each day) and warm temperatures during the growing season are needed. Factors such as fog, wind, elevation, aspect (north- or south-facing slope), and distance from the ocean affect sunlight level and temperature. Keep in mind:

- Summer temperatures increase as the distance from the coast increases.

- Heat levels are greater near pavement and lightcolored walls.

- Sites toward the bottom of slopes are colder than those at the top (except in summer where higher sites may stay above the fog).

- Summer fog keeps coastal locations too cool in the summer for varieties that require heat (fig. 4).

For some crops, wind protection will be needed. Leaf burn, leaf drop, crown deformation, fruit drop, and fruit scarring can occur in locations exposed to wind, such as near the coast or along hilltops and ridges.

Generally, when choosing sites for temperate zone crops, select locations where it is warmest in the summer, coldest in the winter, and most protected from wind and salt spray. For subtropical crops, select locations where it is warmest in the summer, mildest in the winter, and protected from wind and salt spray. 


\section{All About Chill Hours}

After losing their leaves and entering a state of dormancy in the fall and winter period, temperate zone crops have two requirements to break dormancy: a sufficient number of chill hours and warm spring temperatures. Chill hours are accumulated

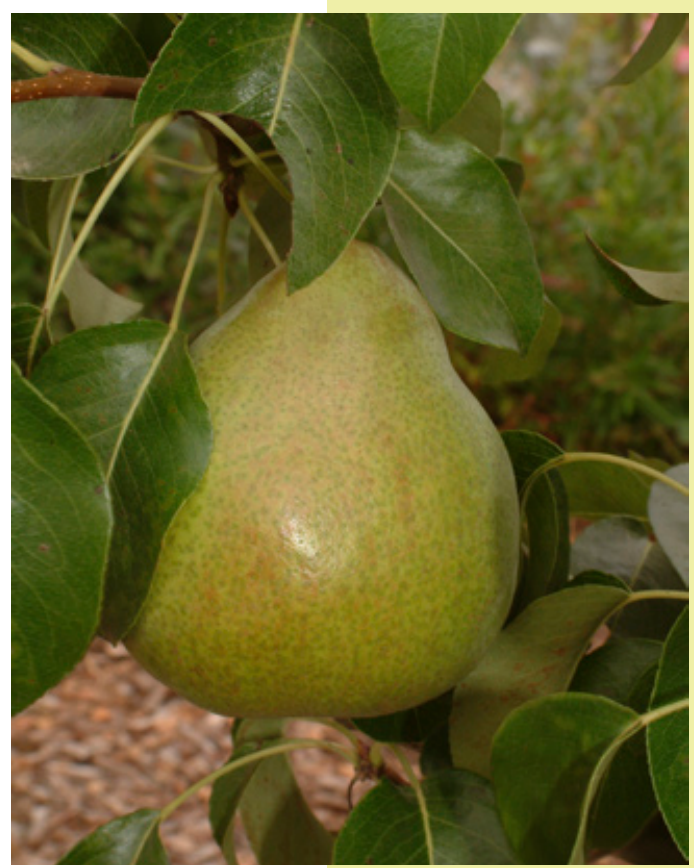
from November through February when temperatures are between $32^{\circ}$ and $45^{\circ} \mathrm{F}$. If chill hour accumulation is insufficient, effects such as delayed foliation, delayed or extended bloom, and reduced fruit set and/or quality can result. Most locations in northern California receive between 800 and 1,500 chill hours. In San Mateo and San Francisco counties, the range can be from 500 hours (or less) in coastal areas to 1,000 (or more) in inland areas. Chill hours do not have to be consecutive, but midwinter heat spells and very sunny days can reverse chilling. Because there are no specialized weather stations that collect chill hour data in San Mateo and San Francisco Counties, the numbers given are estimates.

For temperate zone crops, best production depends on selecting varieties that will receive a sufficient number of chill hours (fig. 5). The number of chill hours required for crops are given in the "Crop and Variety Selection Table" and "Notes on Crops and Varieties." Chill hour requirements are also listed in many nursery catalogs (see "Resources"). Most data sources give fairly consistent chill requirement values for varieties, but because determining the number of chill hours required is not an

Figure 5. Most European pears have medium to high chill requirements and may not be suitable for coastal locations.

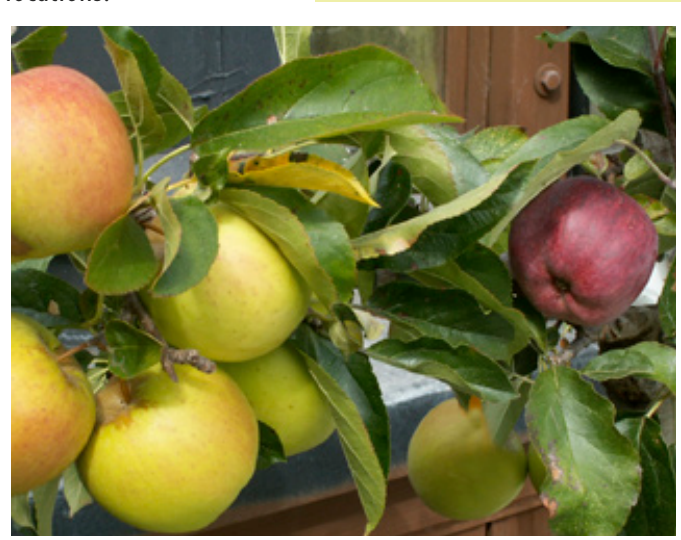

Figure 6. "All-in-one" trees such as this one with three varieties of apples can be a good choice for small spaces.

\section{Tips for Small Spaces}

- Consider using containers. Dwarf varieties are most suitable.

- Try the "3- or 4-in-one" technique where three or four trees are planted in the same hole. Choose varieties that have similar growth habits and can pollinate each other.

- Try "all-in-one" trees where several varieties are grafted onto one tree (fig. 6).

- Create an espalier against a fence or wall.

- Choose a tree with a columnar growth habit such as that of Spire apples.

- Grow grapes or kiwi on a trellis or an arbor.

\section{In Brief}

- Determine your zone: A, B, or C.

- Determine your microclimate factors.

- Choose a variety best suited to your climate.

- Pay attention to pollination requirements.

- Choose varieties that have different harvest times so that everything is not ripe at once. 
Crop and Variety Selection Table

\begin{tabular}{|c|c|c|c|c|c|c|}
\hline $\begin{array}{l}\text { Selected crops and varieties } \\
\text { (Many more varieties are avail- } \\
\text { able that may be suitable. See } \\
\text { "Resources.") }\end{array}$ & $\begin{array}{l}\text { Chill hours } \\
\text { (Data obtained from } \\
\text { nursery catalogs } \\
\text { and other published } \\
\text { sources.) }\end{array}$ & $\begin{array}{l}\text { Self-fruitful } \\
\text { pollination } \\
\text { (Cross-pollination } \\
\text { can increase fruit set } \\
\text { in many self-fruitful } \\
\text { varieties.) }\end{array}$ & $\begin{array}{c}\text { Zone A } \\
600-1,000 \text { chill hours } \\
\text { San Carlos, Redwood City, } \\
\text { Portola Valley, Woodside, } \\
\text { Atherton, East Palo Alto, Menlo } \\
\text { Park, La Honda }\end{array}$ & $\begin{array}{c}\text { Zone B } \\
\text { 400-700 chill hours } \\
\text { Millbrae, San Mateo, } \\
\text { Hillsborough, Burlingame, } \\
\text { Belmont, Foster City }\end{array}$ & $\begin{array}{c}\text { Zone } \mathbf{C} \\
<\mathbf{5 0 0} \text { chill hours } \\
\text { San Francisco, S. San } \\
\text { Francisco, Brisbane, Colma, } \\
\text { Daly City, San Bruno, } \\
\text { Pacifica, Half Moon Bay, } \\
\text { San Gregorio }\end{array}$ & $\begin{array}{l}\text { Harvest } \\
\text { Early: May-June } \\
\text { Mid: July-Aug } \\
\text { Late: Sept-0ct }\end{array}$ \\
\hline Almond & $200-600$ & - & $\begin{array}{c}\text { yes, plant in areas of minimal } \\
\text { spring frost }\end{array}$ & $\begin{array}{l}\text { marginal, plant in warmest } \\
\text { locations }\end{array}$ & no & late \\
\hline All-In-0ne & $400-500$ & yes & yes & marginal & no & late \\
\hline Ne Plus Ultra & $250-350$ & no & yes & marginal & no & late \\
\hline Nonpareil & 400 & no & yes & marginal & no & late \\
\hline Apple & $100-1,800$ & - & all but very high-chill varieties & $\begin{array}{l}\text { moderate- and low-chill } \\
\text { varieties }\end{array}$ & low-chill varieties & - \\
\hline Anna & $200-300$ & yes & yes & yes & yes & early \\
\hline Beverly Hills & 300 & yes & yes & yes & yes & mid \\
\hline Fuji & 400 & yes & yes & yes & yes & late \\
\hline Gala & 500 & yes & yes & yes & yes & mid \\
\hline Golden Delicious & $600-700$ & yes & yes & yes & no & mid-late \\
\hline Granny Smith & 400 & yes & yes & yes & yes & late \\
\hline Gravenstein & 700 & no & yes & yes & no & early-mid \\
\hline Gordon & $300-500$ & yes & yes & yes & yes & mid-late \\
\hline Jonathan & 800 & yes & yes & no & no & mid-late \\
\hline Liberty & 800 & yes & yes & no & no & mid-late \\
\hline Newtown & 700 & yes & yes & yes & no & late \\
\hline Rome Beauty & 700 & yes & yes & yes & no & late \\
\hline Winesap & $800-900$ & no & yes & no & no & late \\
\hline Winter Banana & $400-500$ & partially & yes & yes & yes & late \\
\hline Apricot & $300-1,000$ & - & $\begin{array}{c}\text { yes, plant in areas of minimal } \\
\text { spring frost }\end{array}$ & $\begin{array}{c}\text { yes, plant in areas of minimal } \\
\text { spring frost }\end{array}$ & no & - \\
\hline Blenheim & $400-500$ & yes & yes & yes & no & early-mid \\
\hline Early Golden & 450 & yes & yes & yes & no & early \\
\hline Katy & 350 & yes & yes & yes & no & early \\
\hline Moorpark & $600-700$ & yes & yes & yes & no & mid \\
\hline Aprium & - & - & - & - & - & - \\
\hline Flavor Delight & 300 & $\begin{array}{l}\text { yes but better yields } \\
\text { with any apricot }\end{array}$ & yes & yes & marginal & early \\
\hline Avocado & $\begin{array}{c}0 \\
\text { (subtropical) }\end{array}$ & - & $\begin{array}{l}\text { only in frost-protected } \\
\text { areas }\end{array}$ & $\begin{array}{l}\text { only in frost-protected } \\
\text { areas }\end{array}$ & yes & - \\
\hline Bacon & 0 & yes & yes & yes & yes & late \\
\hline Hass & 0 & yes & yes & yes & yes & late \\
\hline Blueberry & $100-1,800$ & $\begin{array}{l}\text { best yields with more } \\
\text { than one variety }\end{array}$ & yes & yes & marginal, low chill only & - \\
\hline Berkeley & $800-1,000$ & yes & yes & no & no & mid \\
\hline Bluecrop & $800-1,000$ & yes & yes & no & no & mid \\
\hline Earliblue & $800-1,000$ & yes & yes & no & no & early \\
\hline Gulf Coast & $200-300$ & yes & yes & yes & yes & early \\
\hline Jersey & $800-1,000$ & yes & yes & no & no & mid-late \\
\hline Jubilee & $500-700$ & yes & yes & yes & no & early \\
\hline Misty & $150-300$ & yes & yes & yes & yes & mid \\
\hline Revielle & $600-800$ & yes & yes & yes & no & early-mid \\
\hline Cane Berries & $200-800$ & & yes & yes & yes & \\
\hline $\begin{array}{l}\text { Blackberry: } \\
\text { Ollallie, Boysen, Young }\end{array}$ & $200-800$ & yes & yes & yes & yes & mid \\
\hline $\begin{array}{l}\text { Raspberry: } \\
\text { Autumn Bliss, Fairview, } \\
\text { Heritage, Summit }\end{array}$ & $200-800$ & yes & yes & yes & $\begin{array}{l}\text { raspberries are marginal } \\
\text { in very foggy locations }\end{array}$ & mid \\
\hline Cherry & $400-1,400$ & - & $\begin{array}{c}\text { sweet: yes } \\
\text { sour: plant in coldest winter areas }\end{array}$ & moderate-chill varieties only & no & - \\
\hline $\begin{array}{l}\text { Bing, Black Tartarian, } \\
\text { Ranier, Van }\end{array}$ & $700-900$ & no & yes & no & no & early \\
\hline Stella & $700-800$ & yes & yes & no & no & early \\
\hline Montmorency (sour) & $700-1,000$ & no & yes & no & no & early \\
\hline English Morello & $400-700$ & yes & yes & yes & no & early \\
\hline
\end{tabular}




\section{Crop and Variety Selection Table (continued)}

\begin{tabular}{|c|c|c|c|c|c|c|}
\hline $\begin{array}{l}\text { Selected crops and varieties } \\
\text { (Many more varieties are avail- } \\
\text { able that may be suitable. See } \\
\text { "Resources.") }\end{array}$ & $\begin{array}{l}\text { Chill hours } \\
\text { (Data obtained from } \\
\text { nursery catalogs } \\
\text { and other published } \\
\text { sources.) }\end{array}$ & $\begin{array}{c}\text { Self-fruitful } \\
\text { pollination } \\
\text { (Cross-pollination } \\
\text { can increase fruit set } \\
\text { in many self-fruitful } \\
\text { varieties.) }\end{array}$ & $\begin{array}{c}\text { Zone A } \\
600-1,000 \text { chill hours } \\
\text { San Carlos, Redwood City, } \\
\text { Portola Valley, Woodside, } \\
\text { Atherton, East Palo Alto, Menlo } \\
\text { Park, La Honda }\end{array}$ & $\begin{array}{c}\text { Zone B } \\
\text { 400-700 chill hours } \\
\text { Millbrae, San Mateo, } \\
\text { Hillsborough, Burlingame, } \\
\text { Belmont, Foster City }\end{array}$ & $\begin{array}{c}\text { Zone } \mathbf{C} \\
<\mathbf{5 0 0} \text { chill hours } \\
\text { San Francisco, S. San } \\
\text { Francisco, Brisbane, Colma, } \\
\text { Daly City, San Bruno, } \\
\text { Pacifica, Half Moon Bay, } \\
\text { San Gregorio }\end{array}$ & $\begin{array}{l}\text { Harvest } \\
\text { Early: May-June } \\
\text { Mid: July-Aug } \\
\text { Late: Sept-0ct }\end{array}$ \\
\hline Chestnut & $300-500$ & - & yes & yes & yes & - \\
\hline Colossal & $400-500$ & no & yes & yes & yes & late \\
\hline Nevada & $400-500$ & no & yes & yes & yes & late \\
\hline Citrus & $\begin{array}{c}0 \\
\text { (subtropical) }\end{array}$ & yes & $\begin{array}{c}\text { most citrus can be grown, but, } \\
\text { grapefruit and other large fruit } \\
\text { may not sweeten; } \\
\text { may need frost protection }\end{array}$ & $\begin{array}{l}\text { may need frost protection; } \\
\text { most areas not suitable for } \\
\text { grapefruit and other large } \\
\text { fruit }\end{array}$ & $\begin{array}{l}\text { yes for lemons, limes, and } \\
\text { mandarins }\end{array}$ & variable \\
\hline Currant & $800-1,500$ & yes & yes & marginal & no & mid \\
\hline Fig & 100 or fewer & - & yes & yes & yes & late \\
\hline $\begin{array}{l}\text { Black Mission, } \\
\text { White Genoa, Brown Turkey }\end{array}$ & & yes & yes & yes & yes & late \\
\hline Filbert/Hazelnut & $800-1,600$ & - & yes & no & no & - \\
\hline Barcelona & 800 & no & & no & no & late \\
\hline Du Chilly & 800 & no & & no & no & late \\
\hline Gooseberry & $800-1,500$ & yes & yes & marginal & no & mid \\
\hline Grape & $100-500$ & - & yes, may need frost protection & $\begin{array}{l}\text { marginal, winters may be too } \\
\text { mild and summers too cool }\end{array}$ & $\begin{array}{l}\text { no, except for some inland } \\
\text { canyons along the coast }\end{array}$ & late \\
\hline $\begin{array}{l}\text { Wine: } \\
\text { Barbera, Cabernet Sauvignon, } \\
\text { Chardonay, Chenin Blanc, } \\
\text { Gewurztraminer, Grey Riesling, } \\
\text { Merlot, Petite Sirah, } \\
\text { Sangiovese, Sauvingnon Blanc, } \\
\text { White Riesling, Zinfandel }\end{array}$ & 100 & yes & yes & $\begin{array}{l}\text { yes, but may not ripen in cool } \\
\text { summers }\end{array}$ & $\begin{array}{l}\text { no, except for some inland } \\
\text { canyons along the coast }\end{array}$ & mid-late \\
\hline $\begin{array}{l}\text { Table: } \\
\text { California Concord, } \\
\text { Perlette, Cardinal, } \\
\text { Beauty Seedless, } \\
\text { Flame Seedless, } \\
\text { Niagra, Thompson Seedless }\end{array}$ & 100 & yes & yes & $\begin{array}{l}\text { yes, but may not ripen in cool } \\
\text { summers }\end{array}$ & $\begin{array}{l}\text { no, except for some inland } \\
\text { canyons along the coast }\end{array}$ & late \\
\hline Guava & - & - & - & - & - & - \\
\hline Pineapple (Feijoa) & 50 & no & yes & yes & marginal & late \\
\hline Coolidge & 50 & yes & yes & yes & marginal & \\
\hline $\begin{array}{l}\text { Strawberry (true } \\
\text { guava) }\end{array}$ & $\begin{array}{c}0 \\
\text { (subtropical) }\end{array}$ & no & $\begin{array}{l}\text { marginal, needs frost } \\
\text { protection }\end{array}$ & marginal & no & early-late \\
\hline Kiwi & $50-850$ & - & yes, may need frost protection & $\begin{array}{l}\text { yes, may need frost } \\
\text { protection }\end{array}$ & $\begin{array}{l}\text { yes, in warmest areas; wind } \\
\text { protection may be needed }\end{array}$ & - \\
\hline Fuzzy: Hayward & $600-800$ & no & yes & yes & $\begin{array}{l}\text { yes, in warmest areas; wind } \\
\text { protection may be needed }\end{array}$ & late \\
\hline Chico & $600-800$ & no & yes & yes & $\begin{array}{l}\text { yes, in warmest areas; wind } \\
\text { protection may be needed }\end{array}$ & late \\
\hline Smooth: Ken's Red & undetermined & no & yes & yes & yes & late \\
\hline Kolomikta & undetermined & no & yes & yes & yes & late \\
\hline Loquat & 0 & yes & yes & yes & yes & early \\
\hline Lychee & $100-200$ & yes & marginal & marginal & marginal & early-mid \\
\hline Mulberry & $400-450$ & - & yes & yes & yes & early \\
\hline Persian fruiting & $400-450$ & yes & yes & yes & yes & early-mid \\
\hline White fruiting & $400-450$ & yes & yes & yes & yes & early-mid \\
\hline Nectarine & $100-1,200$ & - & yes & yes & marginal & - \\
\hline Fantasia & 500 & yes & yes & yes & marginal & mid \\
\hline Flame Kist & 700 & yes & yes & yes & no & mid-late \\
\hline Flavortop & $600-700$ & yes & yes & yes & no & mid \\
\hline Goldmine & 400 & yes & yes & yes & marginal & mid \\
\hline Independence & $700-800$ & yes & yes & no & no & early-mid \\
\hline Panamint & $250-300$ & yes & yes & yes & marginal & mid-late \\
\hline Snow Queen & $250-300$ & yes & yes & yes & marginal & early-mid \\
\hline Olive & $100-200$ & - & yes & yes & $\begin{array}{l}\text { no, except in warmest } \\
\text { areas }\end{array}$ & - \\
\hline Manzanillo & $100-200$ & yes & yes & yes & marginal & late \\
\hline Mission & $100-200$ & yes & yes & yes & marginal & late \\
\hline Sevillano & $100-200$ & yes & yes & yes & marginal & late \\
\hline
\end{tabular}




\section{Crop and Variety Selection Table (continued)}

\begin{tabular}{|c|c|c|c|c|c|c|}
\hline $\begin{array}{l}\text { Selected crops and varieties } \\
\text { (Many more varieties are avail- } \\
\text { able that may be suitable. See } \\
\text { "Resources.") }\end{array}$ & $\begin{array}{l}\text { Chill hours } \\
\text { (Data obtained from } \\
\text { nursery catalogs } \\
\text { and other published } \\
\text { sources.) }\end{array}$ & $\begin{array}{c}\text { Self-fruitful } \\
\text { pollination } \\
\text { (Cross-pollination } \\
\text { can increase fruit set } \\
\text { in many self-fruitful } \\
\text { varieties.) }\end{array}$ & $\begin{array}{c}\text { Zone A } \\
600-1,000 \text { chill hours } \\
\text { San Carlos, Redwood City, } \\
\text { Portola Valley, Woodside, } \\
\text { Atherton, East Palo Alto, Menlo } \\
\text { Park, La Honda }\end{array}$ & $\begin{array}{c}\text { Zone B } \\
400-700 \text { chill hours } \\
\text { Millbrae, San Mateo, } \\
\text { Hillsborough, Burlingame, } \\
\text { Belmont, Foster City }\end{array}$ & $\begin{array}{c}\text { Zone } \mathbf{C} \\
<\mathbf{5 0 0} \text { chill hours } \\
\text { San Francisco, S. San } \\
\text { Francisco, Brisbane, Colma, } \\
\text { Daly City, San Bruno, } \\
\text { Pacifica, Half Moon Bay, } \\
\text { San Gregorio }\end{array}$ & $\begin{array}{l}\text { Harvest } \\
\text { Early: May-June } \\
\text { Mid: July-Aug } \\
\text { Late: Sept-Oct }\end{array}$ \\
\hline Peach & $100-1200$ & - & yes & yes & marginal & - \\
\hline (white) Babcock & $250-400$ & yes & yes & yes & marginal & mid \\
\hline Desert Gold & $250-400$ & yes & yes & yes & marginal & early \\
\hline Elbertas & $700-850$ & yes & yes & no & no & mid \\
\hline Flavorcrest & 750 & yes & yes & no & no & mid \\
\hline Forty niner & $700-800$ & yes & yes & no & no & mid \\
\hline Frost & 700 & yes & yes & yes & no & mid \\
\hline (white) Nectar & $800-1,000$ & yes & yes & no & no & mid \\
\hline Redhaven & $800-950$ & yes & yes & no & no & mid \\
\hline (donut) Saturn & $250-300$ & yes & yes & yes & marginal & mid \\
\hline Suncrest & 700 & yes & yes & yes & no & mid-late \\
\hline Pear & $200-1,500$ & - & yes & yes & $\begin{array}{l}\text { low-chill and Asian } \\
\text { varieties best }\end{array}$ & - \\
\hline Asian: Hosui & 450 & yes & yes & yes & yes & mid \\
\hline Shinseiki & $350-450$ & yes & yes & yes & yes & mid \\
\hline Twentieth Century & 400 & yes & yes & yes & yes & mid \\
\hline European: Bartlett & 800 & yes & yes & yes & no & mid \\
\hline Bosc & $700-800$ & no & yes & no & no & mid-late \\
\hline Comice & $200-600$ & yes & yes & yes & marginal & late \\
\hline D'Anjou & 800 & no & yes & no & no & late \\
\hline Seckel & $300-800$ & yes & yes & yes & marginal & late \\
\hline Winter Nelis & 700 & no & yes & yes & no & late \\
\hline Persimmon & $\approx 100-400$ & - & yes & yes & yes & - \\
\hline Chocolate & $100-200$ & yes & yes & yes & yes & late \\
\hline Fuyu & $100-200$ & yes & yes & yes & yes & late \\
\hline Hachiya & $100-200$ & yes & yes & yes & yes & late \\
\hline Plum & $250-1,100$ & - & yes & yes & yes & mid \\
\hline Burgundy & $250-400$ & yes & yes & yes & yes & mid \\
\hline Damson & 800 & yes & yes & yes & no & mid \\
\hline Friar & 400 & yes & yes & yes & yes & mid \\
\hline Green Gage & $300-600$ & yes & yes & yes & yes & mid \\
\hline Kelsey & 400 & no & yes & yes & yes & mid \\
\hline Laroda & 400 & no & yes & yes & yes & mid \\
\hline Nubiana & $400-500$ & yes & yes & yes & yes & mid \\
\hline Queen Anne & 500 & no & yes & yes & yes & mid \\
\hline Santa Rosa & $300-400$ & yes & yes & yes & yes & early \\
\hline Satsuma & 300 & no & yes & yes & yes & mid \\
\hline Wickson & 500 & no & yes & yes & yes & mid \\
\hline Pluot & $400-800$ & - & - & - & - & mid \\
\hline Dapple Dandy & $400-500$ & no & yes & yes & marginal & mid \\
\hline Pomegranate & $50-300$ & - & yes & yes & no & mid-late \\
\hline Wonderful & 150 & yes & yes & yes & no & late \\
\hline Quince & $100-500$ & - & yes & yes & low chill & mid \\
\hline Pineapple & $100-300$ & yes & yes & yes & yes & late \\
\hline Strawberry & $50-300$ & - & yes & yes & yes & - \\
\hline $\begin{array}{l}\text { Short-day: } \\
\text { Chandler Pajaro, Tioga }\end{array}$ & $200-300$ & yes & yes & yes & yes & early \\
\hline $\begin{array}{l}\text { Day-neutral: Aptos, } \\
\text { Fern, Seascape, Sequoia }\end{array}$ & $200-300$ & yes & yes & yes & yes & early-late \\
\hline Walnut & $300-1,500$ & - & yes & marginal & no & - \\
\hline Hartly & $500-700$ & yes & yes & yes & no & late \\
\hline Chandler & $700-1,000$ & yes & yes & no & no & late \\
\hline Idaho & $300-700$ & yes & yes & yes & no & late \\
\hline Payne & 500 & no & yes & yes & no & late \\
\hline
\end{tabular}




\section{NOTES ON CROPS AND VARIETIES}

\section{Almond}

Chill hours: 250 to 500

Almonds are stone fruits eaten as nuts. They bloom early (February and March) when the weather is cold and rainy, and thus are susceptible to poor pollination and to frost (in the colder area of the counties). A long, dry summer (180 to 240 days) is required for the nuts to mature, and summer heat is required to develop best flavor. Cross-pollination is required for most varieties. Ne Plus Ultra is a good pollenizer.

\section{Apple}

Chill hours: 100 to 1,800

There are hundreds of apple varieties to choose from. Most apples require cross-pollination. Some varieties, such as Golden Delicious and Yellow Newtown, are partially self-fruitful and will usually set a moderate crop without another pollenizer. Others, such as Gravenstein and Winesap, have sterile pollen. Gala and Fuji are good pollenizers. Where space is limited, multiple variety (all-in-one) trees are available in standard or dwarf sizes. Choose varieties for taste, purpose (cooking or eating fresh), disease resistance, and chill requirements. Codling moth is the most likely reason for wormy apples (see UC IPM Pest Note Codling Moth, ANR Publication 7412.) For a list including this and other Pest Notes, see the "Resources" section below.

\section{Apricot}

Chill hours: 300 to 1,000

Apricots bloom early and are subject to the same frost and poor pollination problems as almonds if the early spring weather is cold and rainy. Most are self-fruitful and can be harvested 100 to 120 days from full bloom.

\section{Aprium}

Chill hours: fewer than 300

Aprium is a hybrid between apricot and plum. Flavor Delight is self-fruitful, but gets better yields when pollenized by any apricot.

\section{Avocado}

Chill hours: none required

Avocado is most productive in full sun but can be grown in all three zones. Mexican varieties are least likely to be damaged by frost. It can become a large tree and is subject to root rot in poorly drained soil. Trees grown from seed can take up to 12 years to produce fruit. Trees sold in nurseries have been vegetatively propagated and are productive sooner.

\section{Blueberry}

Chill hours: 100 to 1,000

Blueberries perform best in acidic soil. Highbush varieties (up to 6 to $8 \mathrm{ft}$ tall by $3 \mathrm{ft}$ wide, or 1.8 to $2.4 \mathrm{~m}$ tall by $0.9 \mathrm{~m}$ wide) are best adapted to California climate zones. Some varieties, such as Misty, Sunshine Blue, and Toro, also have outstanding landscape characteristics such as showy flowers and colorful foliage. California huckleberries are delicious close relatives of eastern blueberries, and can be found in moist, wooded, wildland areas in San Mateo County. 


\section{Cane Berries}

Chill hours: 200 to 800

Blackberry

Blackberries are adapted to all three zones. Varieties include Boysen, Ollalie, Logan, and Young. Native California blackberry (Rubus ursinus), and the introduced

Himalyan blackberry (Rubus discolor), are commonly found in moist wildland and disturbed areas in San Mateo county.

Raspberry

Raspberries are best adapted to zones A and B. Varieties vary in color (red, yellow, black, purple), flavor, and harvest times (summer or fall).

\section{Cherry}

Chill hours: 400 to 1,400

Sour cherries have high chill requirements and are self-fruitful. Almost all sweet cherry varieties require cross- pollination. Stella and English Morello are sweet varieties that are self-fruitful. Black Tartarian is a good pollenizer for most varieties. Cherry trees tend to be susceptible to root rot in poorly drained soil.

\section{Chestnut}

Chill hours: 300 to 500

Because of low chilling requirements, chestnuts could be grown in all three zones; however, little research has been done in California about specific adaptability or productive capacity. Cross-pollination is required. It can become a very large tree (up to $80 \mathrm{ft}$, or $240 \mathrm{~m}$, tall), so adequate growing space should be a primary consideration. Litter and unpleasant-smelling pollen can be a deterrent. Be sure not to confuse the edible chestnut (Castanea spp) with the ornamental horse chestnut or buckeye (Aesculus spp), which contain toxins.

\section{Citrus}

Chill hours: none required

Certain varieties of citrus can be grown in all three zones. In coastal locations where sunny days and warm temperatures are rare in the summer, choose lemons, limes, and mandarins for best results. Grapefruit, pummelo, and other large-fruited varieties require long, hot summers; in northern California climates, compared with hotter locations, they must be left on the tree up to 6 months longer to ripen. Lemons and limes bloom almost all year round (especially in coastal locations), resulting in trees with fruit in several stages of maturity at any one time. Frost protection is required. Citrus fruit and leaves and twigs at the edge of the canopy are most likely to suffer frost damage. Citrus trees are available in dwarf sizes, are suitable for growing in containers, and are an attractive addition to a home landscape.

\section{Currant}

Chill hours: 800 to 1,500

Currant and gooseberry do best in cool, humid, partially shaded areas. Cultivated varieties can be grown for the fruit in San Mateo County's coldest locations, elsewhere for their ornamental value. Most varieties are self-fruitful. 


\section{Fig}

Chill hours: 100 or fewer

Figs are easy to grow, but a full day of sun is best for ripening fruit and the fruit must be allowed to ripen on the tree. In cool, foggy locations, try to plant the fig where it can take advantage of a warm spot and reflected light. Most varieties require no crosspollination. Figs are becoming invasive in some riparian areas because seeds and plant fragments are spread by birds and deer.

\section{Filbert/Hazelnut}

Chill hours: 800 to 1,600

California has its own native filbert (Corylus cornuta var. californica) which can be found in moist, shady, wildland areas in San Mateo County. Cultivated varieties require crosspollination and high chill hours. Barcelona and DuChilly are pollinators for each other.

\section{Gooseberry}

Chill hours: 800 to 1,000

Gooseberry and currant do best in cool, humid, partially shaded areas. California native (Ribes spp.) species can be found in wildland areas in San Mateo County, but are thorny and less palatable than cultivated varieties.

\section{Grape}

Chill hours: 100 to 500

In 2004, 86 acres of wine grapes were being grown commercially in San Mateo County, up from 40 acres in 1999. However, in the years between 1870 and 1915, this was a thriving commercial industry, with wine grapes grown primarily around Woodside, Portola Valley, Menlo Park, and Belmont. Some of the factors that led to the industry's decline in this region included Prohibition, the insect pest "phylloxera," urbanization, and the realization that other areas in California where summers are more consistently hot, such as the Napa Valley, were better suited to viticulture. San Mateo and San Francisco County's proximity to the bay and ocean results in a climate that is less favorable. Still, the home vineyard can be successful, particularly in the warmer areas of San Mateo County, but yields may vary year to year.

California wild grape, Vitis californica, can be found in moist, wildland canyons in San Mateo County. Roger's Red is a cultivated variety that produces brilliant red foliage in the fall.

\section{Guava}

Chill hours: 50 for Feijoa

Guava is native to tropical climate zones and most varieties are subject to cold damage. Strawberry guava or purple strawberry guava are hardier than other true guavas and could be tried in sheltered locations.

Distantly related to true guava, pineapple guava (Feijoa sellowiana) is commonly grown as an ornamental shrub. It has an edible flower and can be grown in all zones. For best fruit production, select a named variety and plant where summer temperatures reach $80^{\circ} \mathrm{F}\left(26.7^{\circ} \mathrm{C}\right)$. Some varieties, such as Coolidge, are self-fruitful, but all produce best with cross-pollination. 


\section{Kiwi}

Chill hours: 50 to 850

Kiwi is frost sensitive, especially when young. Summer heat is a requirement for best flavor and maturation. Compatible male and female varieties must be planted together for successful pollination. The fuzzy skin variety, Hayward, is grown commercially on the San Mateo coast. Smooth skin varieties are more cold tolerant.

\section{Loquat}

Chill hours: none required

Loquat trees are more commonly grown in San Mateo and San Francisco counties for their ornamental value than for fruit, which is often considered a nuisance. For best fruit, choose a white-fleshed variety for coastal locations and orange-fleshed varieties for inland locations. Some varieties are self-fruitful.

\section{Lychee}

Chill hours: 100 to 200 hours

The San Francisco Bay Area climate is not ideal for growing lychee for abundant fruit production. It requires cool winters (but is subject to frost damage) with low rainfall and warm, humid summers for best fruit production. It can be grown ornamentally, however, and is especially attractive when in flower and as the red fruit ripens.

\section{Mulberry}

\section{Chill hours: 400 to 450}

The mulberry tree that is most commonly seen in the San Francisco Bay Area is fruitless white mulberry (Morus alba). It can reach 60 feet tall $(18 \mathrm{~m})$ with large surface roots. Varieties grown for fruit have similar growth characteristics, although black varieties are smaller. If you choose to grow a variety for fruit, be sure to give it plenty of room in a location where fruit drop will not be a problem.

\section{Nectarine}

Chill hours: 100 to 1,200

Nectarine is a peach without the fuzzy skin. It is self-fruitful. A few low-chill varieties are available, but they are still not appropriate for Zone $C$ unless a warm location out of the summer fog can be found. Peach leaf curl is a very common fungus disease, especially where wet conditions occur in the spring (see UC IPM Pest Note Leaf Curl, ANR Publication 7426).

\section{Olive}

Chill hours: 100 to 200

Olive trees are grown ornamentally in all three zones. Best fruit production occurs where summers are hot and dry. Before planting an olive for fruit production, consider that the insect pest "olive fruit fly" is not easily controlled in home orchards (see UC IPM Pest Note Olive Fruit Fly, ANR Publication 74112).

\section{Peach}

Chill hours: 100 to 1,200

Peaches and nectarines are self-fruitful A few low-chill varieties are available, but they are still not appropriate for Zone $C$ unless a warm location out of the summer fog can be found. Peach leaf curl is a very common fungus disease, especially where wet conditions occur in the spring (see UC IPM Pest Note Leaf Curl, ANR Publication 7426). Frost is a variety resistant to leaf curl. 


\section{Pear}

Chill hours: 200 to 1,500

Pear trees are vigorous growers with upright branches and need careful pruning to keep to a manageable size. Fire blight is a common problem (see UC IPM Pest Note Fire Blight, ANR Publication 7414). Resistant varieties are available. Some varieties are self-fruitful, but yields are better with cross-pollination. Asian varieties such as Hosui require fewer chill hours than European varieties.

\section{Persimmon}

Chill hours: 100 to 400

Persimmons do well in all zones. Some varieties are self-fruitful. Astringent varieties are unpalatable until they soften. Nonastringent varieties can be eaten when crisp. Unless nonastringent varieties receive sufficient summer heat, however, some astringency may remain even though the fruit is ripe.

\section{Pluot}

Chill hours: 400 to 800

Pluot is a hybrid between plum (three-quarters) and apricot (one-quarter). Where the mix is 50-50 apricot and plum, the fruit is called a plumcot. Pollinators, such as Santa Rosa, are required.

\section{Plum}

Chill hours: 250 to 1,100

Plums do well in all three zones. Japanese varieties are recommended because most have low to moderate chill requirements. Some are self-fruitful. European varieties that can be dried are called prunes.

\section{Pomegranate}

Chill hours: 50 to 300

Pomegranates are adapted to climates with cool winters and hot summers. They are self-fruitful, but cross-pollination increases the fruit set. Fluctuation of soil moisture levels when the fruit is maturing can cause fruit to split.

\section{Quince}

Chill hours: 100 to 500

Quince is typically grown not for its fruit but for its ornamental value, because the fruit is more palatable cooked than eaten fresh. Several varieties are available, some with low chill requirements, and all are self-fruitful.

\section{Strawberry}

Chill hours: 100 to 300

Strawberries grow best with 6 hours per day of sun during the growing season and with winter soil temperatures averaging $55^{\circ} \mathrm{F}\left(12.8^{\circ} \mathrm{C}\right)$ or less. Choose between shortday and day-neutral varieties. The former produces a large crop in late spring and the latter bears continuously during the growing season.

\section{Walnut}

Chill hours: 300 to 1,500

In the home garden, a walnut tree can be a handsome, large shade tree up to 50 feet $(20 \mathrm{~m})$ or more in height. Walnuts are self-fruitful and wind-pollinated, but planting two varieties can improve fruit set. Late-leafing varieties like Chandler and Hartly are more trouble free. Northern California black walnut (Juglans hindsii) is native to the San Francisco Bay Area and can be found in scattered canyon and valley locations. The nut is flavorful but difficult to remove from the shell. 


\section{RESOURCES}

\section{Internet}

\section{The University of California Research and Information Centers}

Research-based information is provided via the internet for researchers, industry, government agencies, and the general public at http://rics.ucdavis.edu.

One of the UC information centers is the Fruit and Nut Information Center (http://fruitsandnuts.ucdavis.edu), which provides information on 45 fruits, nuts, and berries.

A part of the Fruit and Nut Information Center is The California Backyard Orchard (http://homeorchard.ucdavis.edu), which is devoted to growing fruits, nuts, and berries at home. It provides information on such topics as plant selection, planting, irrigation, pruning, pests and diseases, and fertilization. Detailed information is provided on almond, apple, apricot, avocado, berries, cherry, chestnut, citrus, fig, filbert, grape (table), kiwi, loquat, nectarine, olive, peach, pear, pecan, persimmon, pistachio, plum and prune, pomegranate, quince, and walnut.

\section{The University of California Agriculture and Natural Resources (ANR) Online Catalog}

This Web site (http://anrcatalog.ucdavis.edu) lists the following free downloadable publications:

Almonds: Calendar of Operations for Home Gardeners, Publication 7257

Apples and Pears: Calendar of Operations for Home Gardeners, Publication 7258

Apricots: Calendar of Operations for Home Gardeners, Publication 7259

Budding and Grafting Citrus and Avocado in the Home Garden, Publication 8001

Cherries: Calendar of Operations for Home Gardeners, Publication 7260

Fruit Trees: Planting and Care of Young Trees, Publication 8048

Fruit Trees: Thinning Young Fruit, Publication 8047

Harvesting and Storing Your Home Orchard's Nut Crop, Publication 8005

Navel Orange Split, Publication 8038

Peaches and Nectarines: Calendar of Operations for Home Gardeners, Publication 7261

Plums: Calendar of Operations for Home Gardeners, Publication 7262

Walnuts: Calendar of Operations for Home Gardeners, Publication 7263

\section{UC IPM Pest Notes}

This is a series of single-subject publications on pest and disease management that can also be downloaded free from the ANR Catalog Web site above. The following are relevant to home orchard pest management:

Aphids, Publication 7404

Apple Scab, Publication 7413

Coddling Moth, Publication 7412

Fireblight, Publication 7414

Fruit Tree Leaf Roller, Publication 7473

Glassy-Winged Sharpshooter, Publication 7492
Leaf Curl, Publication 7426

Olive Fruit Fly, Publication 74112

Powdery Mildew on Fruits and Berries, Publication 7494

Scales, Publication 7408

Walnut Husk Fly, Publication 7430

If you do not have access to the internet and would like one of the above publications to be mailed to you, call the San Mateo-San Francisco County Cooperative Extension Office at 650-726-9059.

\section{The California Rare Fruit Growers}

This organization (http://www.crfg.org) has chapters all around California. Its members share information about growing fruit (rare and common varieties), but with an emphasis on subtropical and tropical varieties. 


\section{Publications}

\section{Of Special Interest}

California Master Gardener Handbook (ANR Publication 3382) devotes separate chapters to grapes, berries, temperate tree fruit and nut crops, citrus, and avocados. The handbook covers the topics extensively and includes information about growth and development, varieties, rootstocks, pruning and more. The chapters were developed assuming no prior knowledge on the part of the reader.

The Home Orchard: Growing Your Own Deciduous Fruit and Nut Trees (ANR Publication 3485) is an all-in-one manual for pome fruit, stone fruit, and nuts as well as fig and persimmon. Chapters, with photos and illustrations in color, cover all aspects of home orchard management from planting to harvest, including a crop-by-crop, month-by-month calendar of cultural practices.

Pests of the Garden and Small Farm: A Grower's Guide to Using Less Pesticide (ANR Publication 3332) is available through the ANR Catalog Web site above and covers the subject of pest and disease management in great detail. It is designed to help gardeners protect their crops from pests with minimum use of pesticides. Color photographs and comparison tables can aid in problem diagnosis.

\section{Further Reading}

Brenzel, K. N., ed. 2007. Sunset western garden book, 8th ed. Menlo Park, CA: Sunset Books.

California Rare Fruit Growers. Fruit facts, Vols. 1 and 2. California Rare Fruit Growers Web site, http://www.cfrg.org.

Vossen, P. M. and D. Silver. 2000. Growing temperate tree fruit and nut crops in the home garden. University of California Research and Information Center, The California Backyard Orchard Web site, http://homeorchard.ucdavis.edu.

Walheim, L. 1996. Citrus: The complete guide to selecting and growing more than 100 varieties for California, Arizona, Texas and the Gulf Coast. Tucson, AZ: Ironwood Press.

\section{Nurseries}

Several wholesale and retail nurseries specialize in fruit, nut, and berry production. Some offer mail order or internet options. Catalogs and Web sites show color photos and detailed descriptions of the varieties offered. If the variety you are looking for is offered at a wholesale-only nursery, ask your local retail nursery to order it for you.

The following wholesale and retail nurseries offer color photos, chill hour and pollination requirements, root stock information, harvest times, and descriptions of color and flavor.

Bay Laurel Nursery (retail)

Atascadero, CA

805-466-3406

http://www.baylaurelnursery.com

Dave Wilson Nursery (wholesale)

Hickman, CA

209-874-1821

http://www.davewilson.com

Four Winds Growers (wholesale citrus)

Fremont, CA

510-656-2591

http://www.fourwindsgrowers.com
L.E. Cooke Nursery (wholesale)

Visalia, CA

209- 732-9146

http://www.lecooke.com

Peaceful Valley Farm Supply (retail)

Grass Valley, CA

530-272-4769

http://www.groworganic.com

Raintree Nursery (retail)

Moreton, WA

360-496-6400

http://www.raintreenursery.com 


\section{FOR MORE INFORMATION}

To order or obtain printed ANR publications and other products, visit the ANR

Communication Services online catalog at http://anrcatalog.ucdavis.edu. You can also place orders by mail, phone, or FAX, or request a printed catalog of our products from:

University of California

Agriculture and Natural Resources

Communication Services

6701 San Pablo Avenue, 2nd Floor

Oakland, California 94608-1239

Telephone: (800) 994-8849 or (510) 642-2431

FAX: (510) 643-5470

E-mail inquiries: danrcs@ucdavis.edu

An electronic version of this publication is available on the ANR Communication Services Web site at http://anrcatalog.ucdavis.edu.

\section{Publication 8261}

This publication is a revised version of Laurence R. Costello, Warren C. Micke, and James A. Beutel, Fruit, Nut and Grape Varieties for Home Orchards: San Mateo and San Francisco Counties (Oakland: University of California Division of Agriculture and Natural Resources, Publication 21338, 1983).

ISBN-13: 978-1-60107-482-9

(C) 2007 The Regents of the University of California, Division of Agriculture and Natural Resources. All rights reserved.

To simplify information, trade names of products have been used. No endorsement of named or illustrated products is intended, nor is criticism implied of similar products that are not mentioned or illustrated.

The University of California prohibits discrimination or harassment of any person on the basis of race, color, national origin, religion, sex, gender identity, pregnancy (including childbirth, and medical conditions related to pregnancy or childbirth), physical or mental disability, medical condition (cancer-related or genetic characteristics), ancestry, marital status, age, sexual orientation, citizenship, or status as a covered veteran (covered veterans are special disabled veterans, recently separated veterans, Vietnam era veterans, or any other veterans who served on active duty during a war or in a campaign or expedition for which a campaign badge has been authorized) in any of its programs or activities. University policy is intended to be consistent with the provisions of applicable State and Federal laws.

Inquiries regarding the University's nondiscrimination policies may be directed to the Affirmative Action/Staff Personnel Services Director, University of California, Agriculture and Natural Resources, 1111 Franklin Street, 6th Floor, Oakland, CA 94607-5201, (510) 987-0096. For a free catalog of other publications, call (800) 994-8849. For help downloading this publication, call (530) 297-4445.

This publication has been anonymously peer reviewed for technical accuracy by University of California scientists and other qualified professionals. This review process was managed by the ANR Associate Editor for Pomology, Viticulture, and Subtropical Horticulture.

To simplify information, trade names of products have been used. No endorsement of named or illustrated products is intended, nor is criticism implied of similar products that are not mentioned or illustrated.

pr-6/07-LR/CM 